\title{
Thermodynamics of Twinning
}

I. Müller and G. Zak*

Technische Universität Berlin, 10623, Berlin, Germany

* National Technical University, 252056, Kiev, Ukraine

\begin{abstract}
The quasiplastic hysteresis occuring in shape memory alloys is associated with twinning in tension and compression, i.e. a conversion of one martensitic variant into antoher one. It shows the phenomena of internal elasticity and internal yield, heretofore studied extensively in pseudoelasticity, e.g. see [1], [2]. This paper presents experimental evidence of such phenomena and it offers partial explanation in identifying the onset of yield as an instability phenomenon due to coherency. That conjecture is motivated from a previous consideration of pseudoelasticity.
\end{abstract}

\section{TWINNING IN TENSION AND COMPRESSION. PHENOMENA AND SCHEMATIC VIEW}

At low temperatures a shape memory alloy exhibits what has been called „quasiplastic“ behaviour in a tension-compression test, see Fig. 1.

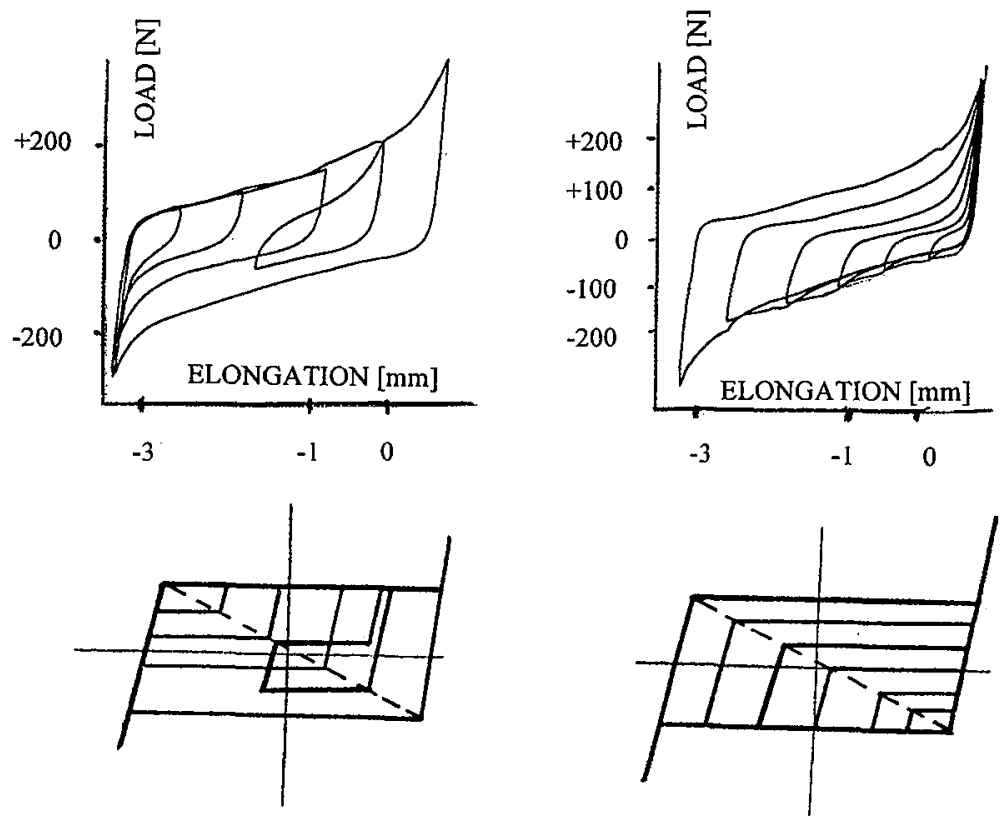

Figure 1: The quasiplastic hysteresis loop, top: measured, bottom: scheriatic.

Measurements with a Cu-12,4 Al-4,5 $\mathrm{Mn}(\mathrm{wt} \%)$ single crystal at $\mathrm{T}=30^{\circ} \mathrm{C}$. 
The flat parts of the measured curves are due to martensitic twinning. The curves show a rich behaviour inside the hysteresis loop when the yield processes are interrupted by unloading or reloading: The internal curves contain steep, reversible, elastic branches followed by internal yield line which are more or less parallel to the main outer yield lines that form the upper and lower border of the hysteresis region. There is also one instance of ,return point memory“, a phenomenon observed by Ortin and Planes (e.g. see [3]) in pseudoelasticity. The experimental yield lines are not horizontal, rather there is a degree of strain hardening as the yield proceeds.

In order to make such curves amenable to a thermodynamic treatment we schematize them in the manner shown in the bottom part of Fig. 1. Essentially only the strain-hardening is neglected in this scheme and all lines are straightened out. We assume that the changeover between internal elasticity and internal yield occurs on the dashed line that rans diagonally across the hysteresis loop. That line will therefore be called the "diagonal“.

The main purpose of this paper is to give a thermodynamic interpretation of the diagonal.

\section{THERMODYNAMICS OF TWINNING}

Phenomenologically twinning is like a phase transition and the thermodynamics of phase transitions is based on a non-convex free energy $f$. In the present case we take the free energy to be a double-well function of the deformation $\mathrm{d}$. The load-deformation curve is then a non-monotone function, see Fig. 2. The two increasing parts represent the thermodynamic behaviour of the twins $(-)$ and $(+)$ respectively.

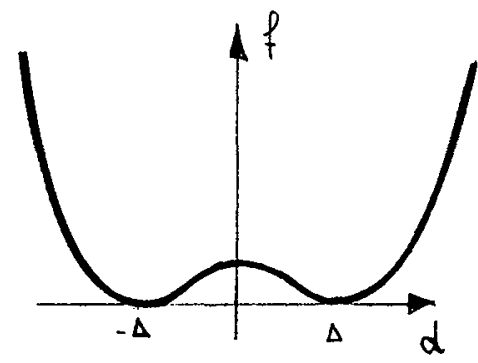

Figure 2: Free energy and load vs. deformation

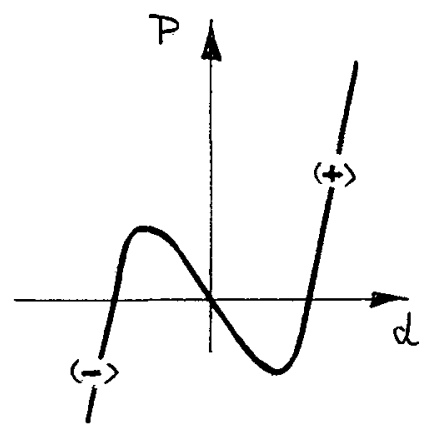

Along the outer yield lines and inside the hysteresis we have a phase mixture so that the total free energy and the total deformation equals

$$
\mathrm{F}=\mathrm{F}_{-}+\mathrm{F}_{+}+\mathrm{Am}-\frac{\mathrm{m}_{+}}{\mathrm{m}} \quad \mathrm{D}=\mathrm{D}_{-}+\mathrm{D}_{+}
$$

or for the specific values with $\mathrm{z}$ as the fraction of the $(+)$ phase

$$
f=(1-z) f\left(d_{-}\right)+z f\left(d_{+}\right)+A z(1-z), \quad d=(1-z) d_{-}+z_{+} .
$$

The last term in the free energy recognizes the contribution of the coherency energy located in the neighbourhoods of phase boundaries. The specific form of this term is the simplest one that conforms to the requirement that it vanish in the pure phases. A is called the coherency coefficient. 
For $d=$ const $f$ tends to a minimum in equilibrium. Necessary conditions for that are given by

$$
\begin{array}{r}
P=\frac{\partial f}{\partial d_{-}}=\frac{\partial f}{\partial d_{+}} \text {and } \begin{array}{r}
P\left(d_{+}-d_{-}\right)=f\left(d_{+}\right)-f\left(d_{-}\right)+A(1-2 z) \\
P\left(d_{+}-d_{-}\right)=\int_{d_{-}}^{d_{+}} P(d) d d+A(1-2 z)
\end{array}
\end{array}
$$

Hence follows that the equilibrium values of $P, d_{-}$and $d_{+}$depend on $z$. These conditions offer themselves for an easy graphical solution in the $(P, d)$-diagram, see Fig. 3 : E.g. for $z=0$ the rectangle $P\left(d_{+}-d_{-}\right)$must be bigger, by $A$, than the integral $\int P d d$ between $d_{-}$and $d_{+}$. This means that the shaded areas must differ by $A$. This determines the load at which phase $(-)$ starts to be in equilibrium with phase $(+)$ and we conjecture that this is the tensile yield load. Accordingly the compressive yield load is constructed by satisfying (2.4) for $z=1$. Thus the outer loop of the hysteresis is constructed as shown in Fig. 3(c). In this way the hysteresis area comes out as equal to $2 \mathrm{~A}$, twice the coherency coefficient.

Equilibrium points for $0<z<1$ are also found by the graphical evaluation of (2.4) and they all lie on a line with negative slope as shown in Fig. 3(d). That line extends diagonally through the hysteresis loop and we conjecture that it represents the diagonal observed in experiments, where the internal yield is triggered.

Since the diagonal slopes downwards the stability of its points may be precarious. We therefore proceed to investigate the question of stability of the phase equilibria.
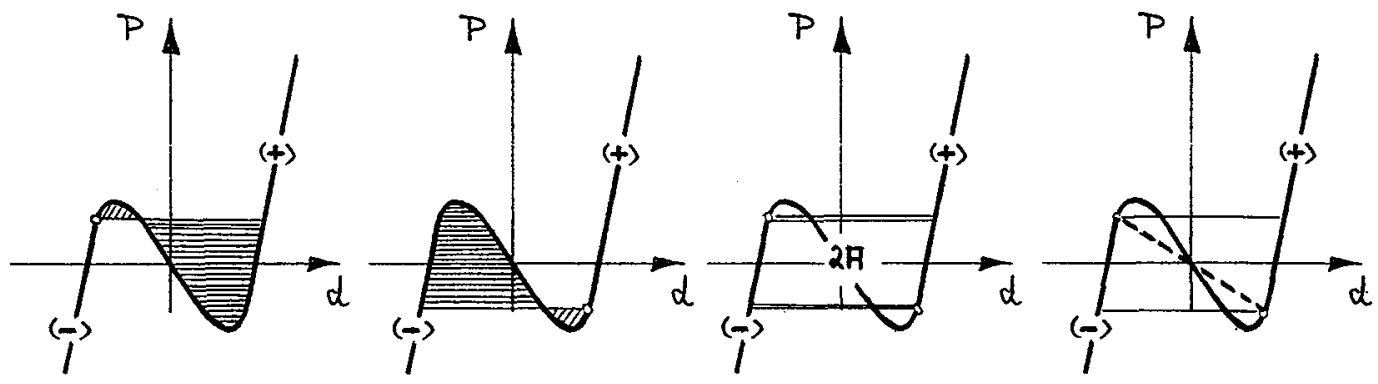

Figure 3: Construction of the hysteresis loop and of the diagonal equilibrium line.

\section{STABILITY OF PHASE EQUILIBRIA}

For simplicity we take the double-well potential to be represented by two parabolae of the form

$$
\mathrm{f}_{ \pm}=\frac{\alpha}{2}(\mathrm{~d} \pm \Delta)^{2} \quad \mathrm{~d}>_{<} 0
$$

We take it that the dynamic equilibrium

$$
P=\left.\frac{\partial \mathrm{f}_{+}}{\partial \mathrm{d}}\right|_{\mathbf{d}_{+}}=\left.\frac{\partial \mathbf{f}_{-}}{\partial \mathrm{d}}\right|_{\mathbf{d}_{-}}
$$


is established, while the phase equilibrium $\frac{\partial f}{\partial z}=0$ is not yet established: In that case we have from (3.1), (3.2)

$$
\mathrm{P}=\alpha\left(\mathrm{d}_{+}-\Delta\right)=\alpha\left(\mathrm{d}_{-}+\Delta\right) \Rightarrow \begin{aligned}
& \mathrm{d}_{-}=\mathrm{d}-2 \Delta \mathrm{z} \\
& \mathrm{d}_{+}=\mathrm{d}-2 \Delta(1-\mathrm{z})
\end{aligned}
$$

and $f$ in (2.2) becomes a function of $d$ and $z$, viz.

$$
f(d, z)=\frac{\alpha}{2}(d+\Delta(1-2 z))^{2}+A z(1-z)
$$

That function has an extremum for

$$
\frac{\partial \mathrm{f}}{\partial \mathrm{z}}=0 \quad \text { hence } \quad \mathrm{z}=\frac{\alpha \Delta}{2 \alpha \Delta^{2}-\mathrm{A}} \mathrm{d}+\frac{1}{2}
$$

and the extremum is a minimum, since

$$
\frac{\partial^{2} f}{\partial z^{2}}=2\left(\alpha \Delta^{2}-A\right)>0
$$

holds. [Note that A must be considered smaller than the elastic energy $\alpha \Delta^{2}$ ].

In the present case the equilibrium line may be explicitly calculated from (3.3) and (3.5). It is given by the function

$$
P=-\frac{\alpha A d}{2 \alpha \Delta^{2}-A}
$$

From (3.6) we conclude that for fixed $d$ the states on this line are stable, since they represent minima of $f$.

On the other hand, if $P$ is fixed, the relevant function, whose extremum determines stability or otherwise, is the free enthalpy $g=f-p d$, or Gibbs free energy. From (2.2) we have for dynamic equilibrium (3.3)

$$
g(P, z)=\frac{P^{2}}{2 \alpha}+A z(1-z)-P\left\{\frac{P}{\alpha}-\Delta(1-2 z)\right\}
$$

This function has an extremum for

$$
\frac{\partial \mathrm{g}}{\partial \mathrm{z}}=0 \quad \text { hence } \quad \mathrm{z}=\frac{1}{2}-\frac{\Delta}{\mathrm{A}} \mathrm{P}
$$

and the extremum is a maximum, since

$$
\frac{\partial^{2} \mathrm{~g}}{\partial \mathrm{z}^{2}}=-2 \mathrm{~A}<0
$$

We conclude that for fixed $\mathrm{P}$ the states on the equilibrium line are unstable, since they represent maxima of $g$. 
Therefore, indeed, the stability on the equilibrium line is precarious: Even though the length of the tensile and compressive specimen, from which the plots of Fig. 1. were taken, is controlled - and not the load -, the internal points may feel that they are under a fixed load. Thus the instability at fixed $\mathbf{P}$ might be more relevant to the body than the stability at fixed $d$. And this fact could be the reason for the onset of internal yield when the diagonal is reached.

\section{OPEN PROBLEMS}

While we think that we have identified the diagonal of Fig. 1 as the line of unstable phase equilibria, many other questions remain open:

- What is the nature of the metastable states on and in the hysteresis loop which are not on the diagonal?

- How do we understand the „return point memory"?

It should be mentioned that analogous questions arise in pseudo-elasticity even though there we do not observe return point memory; rather, in pseudoelasticity, the internal yield continues, once initiated on the diagonal, until the phase transition is complete.

Such questions require the formulation and study of microscopic - or mesoscopic - models. Attempts have been made, e.g. see [4], [5], but so far the success is limited.

\section{References}

[1] Fu, S., Huo, Y., Müller, I., Thermodynamics of pseudoelasticity - an analytical approach, Acta Mechanica 99 (1993) 1-19.

[2] Huo, Y., Müller, I., Non-equilibrium thermodynamics of pseudoelasticity, Cont. Mech. Thermodyn. 5 (1993) 163-204.

[3] J. Ortin, Preisach modeling of hysteresis for a pseudoelastic Cu-Zn-Al single crystal, J. Appl. Phys. 71 (1992) 1454-1461.

[4] Huo, Y., Müller, I., Seelecke, S., Quasiplasticity and pseudoelasticity in shape-memory alloys. In: Phase Transitions and Hysteresis Lecture Notes in Mathematics 1584, A. Visintin (ed). Springer Heidelberg (1994).

[5] Müller, I., Thermodynamik, die Grundlagen der Materialtheorie, Springer Berlin (1994). 